\title{
Resolução temporal: análise em pré-escolares nascidos a termo e pré-termo ${ }^{* * * * *}$
}

\section{Temporal resolution: analysis in term and preterm preschoolers}

\author{
Ana Beatriz Fortes* \\ Liliane Desgualdo Pereira ** \\ Marisa Frasson de Azevedo ***
}

\section{* Fonoaudióloga. Mestre em Ciências pelo Departamento de Fonoaudiologia da Universidade Federal de São Paulo. Endereço para correspondência: Rua Maranduba, 143 - São José dos Campos - SP - CEP 12230-680 (ab.fortes@uol.com.br). \\ **Fonoaudióloga. Livre Docente. Professora Associada do Departamento de Fonoaudiologia da Universidade Federal de São Paulo. \\ ***Fonoaudióloga. Professora Associada e Doutora do Departamento de Fonoaudiologia da Universidade Federal de São Paulo. \\ ****Trabalho Realizado na Universidade Federal de São Paulo.}

Artigo de Pesquisa

Artigo Submetido a Avaliação por Pares

Conflito de Interesse: não

Recebido em 09.06.2005.

Revisado em 21.07.2005; 22.08.2005;

20.04.2006; 16.08.2006; 29.08.2006;

29.09.2006; 28.02.2007.

Aceito para Publicação em 28.02.2007.

\section{Abstract}

Background: auditory processing. Aim: to verify the hearing behavior of temporal resolution in children with ages from five to six years, who were born preterm, with no evidence of neurological alterations and to compare this behavior to that observed in children of the same age, who were born at term, with low risk for developmental disorders, taking into consideration the variables of: threshold detection gaps through preestablished frequency, binaural and monaural presentation, order of stimuli presentation and gender. Method: 70 children divided in two groups: Group 1 with 44 children who were born at term (20 female and 24 male) and Group 2 with 26 preterm children (12 female and 14 male). Children were submitted to audiologic evaluation composed of audiometry, speech response threshold, acoustic impedance test and the Random Gap Detection Test (RGDT). Results: children who were born at term presented lower threshold detection gaps in the RGDT, for both monaural and binaural stimuli presentation, in all of the pre-established frequencies when compared to preterm children. This difference between the groups was statistically significant. The average threshold detection gaps of Group 1 rose according to the increase of frequency. For Group 2 statistically significant differences were not found regarding the average of threshold detection gaps, for both monaural and binaural stimuli presentation. Conclusion: preterm children differ from those born at term regarding the hearing behavior of temporal resolution. The RGDT can be used as a tool to evaluate the hearing process, once the early detection of alterations in temporal processes indicates the need for intervention in order to minimize or avoid future language impairments.

Key Words: Auditory Perception; Hearing Disorders; Infant; Premature.

\section{Resumo}

Tema: processamento auditivo. Objetivo: verificar o comportamento auditivo de resolução temporal de crianças na faixa etária de cinco a seis anos, nascidas pré-termo, sem evidências de alterações neurológicas e compará-lo com o mesmo comportamento auditivo de crianças na mesma faixa etária, nascidas a termo, com baixo risco para alteração do desenvolvimento, considerando as variáveis: limiar de detecção de intervalo de tempo por freqüência sonora pré-estabelecida apresentada na forma binaural e monoaural por ordem de orelha que iniciou o teste e gênero. Método: 70 sujeitos: 44 nascidos a termo reunidos em grupos de 20 indivíduos do sexo feminino e 24 do sexo masculino, denominado Grupo 1, e 26 nascidos pré-termo, sendo 12 indivíduos do sexo feminino e 14 do sexo masculino, denominado Grupo 2, foram submetidos a avaliação audiológica composta por audiometria tonal limiar, limiar de reconhecimento de fala, imitânciometria e aplicação do teste de fusão auditiva denominado de Random Gap Detection. Resultados: os nascidos a termo apresentaram menores limiares de detecção de intervalo de tempo no teste de fusão auditiva, nas formas de apresentação binaural e monoaural em todas as freqüências sonoras pré-estabelecidas, do que os nascidos pré-termo com diferença estatisticamente significante. As médias dos limiares de detecção de intervalo de tempo do Grupo 1 aumentaram conforme a frequiência sonora aumentou. No Grupo 2 não foram encontradas diferenças estatisticamente significantes quanto as médias de limiares de detecção de intervalo de tempo na forma de apresentação binaural e monoaural. Conclusão: os nascidos pré-termo se diferenciam dos nascidos a termo quanto ao comportamento auditivo de resolução temporal e o teste de fusão auditiva utilizado pode servir como ferramenta para a avaliação do processamento auditivo, uma vez que a detecção precoce de alteração dos processos temporais indica uma intervenção para minimizar ou evitar futuros prejuízos de linguagem.

Palavras-Chave: Percepção Auditiva; Transtornos da Audição; Infância; Prematuro.

Referenciar este material como:

1 FORTES, A. B.; PEREIRA, L. D.; AZEVEDO, M. F. de. Resolução temporal: análise em pré-escolares nascidos a termo e pré-termo. Pró-Fono Revista de Atualização $\sum 3$ Científica, Barueri (SP), v. 19, n. 1, p.87-96, jan.-abr. 2007 


\section{Introdução}

A alteração das habilidades auditivas associadas a prejuízos escolares e comportamentais em crianças nascidas pré-termo sem sinais aparentes de déficit neurológico é recentemente relatada na literatura especializada (Dupin et al., 2000; Davis et al., 2001).

A habilidade auditiva de resolução temporal conforme definida pela ASHA (American Speech Hearing Association) em 1996, é um dos aspectos do processamento temporal, que se encontra entre aqueles comportamentos que são denominados de processamento auditivo central. A resolução temporal pode ser definida como a capacidade de detectar intervalos de tempo entre estímulos sonoros ou detectar o menor tempo que um indivíduo possa discriminar entre dois sinais audíveis (Phillips et al., 2000; Shinn, 2003).

Embora as relações entre alterações do processamento auditivo, distúrbio de linguagem e distúrbio de aprendizado seja complexo, a comorbidade é freqüente e particularmente muitas crianças com distúrbio de aprendizagem apresentam alteração no processamento temporal (Cestnik e Jerger, 2000; Bailey e Snowling, 2002; Breir, 2003). O processamento temporal é um dos comportamentos auditivos que tem sido relacionado com a percepção de fala em crianças ouvintes. Especificamente, associam-se alterações do processamento temporal a déficits no processamento fonológico, discriminação auditiva, linguagem receptiva e leitura (Keith, 2000).

Conhecer os testes auditivos que necessitam de respostas comportamentais, atualmente utilizados para avaliar o processamento temporal torna-se imprescindível. Sabe-se que estes testes estão sujeitos a críticas, pois podem falhar em identificar as crianças com e sem problemas. E ainda, há a necessidade de que os avaliadores tenham informações que possam distinguir fatores que comumente confundem a interpretação dos testes tais como falta de motivação, atenção, cooperação e compreensão (Jerger e Musiek, 2000).

Costa (2002) em seu estudo com 35 crianças aplicando o teste de fusão denominado abreviadamente conforme sigla inglesa AFT (Auditory Fusion Test) em tarefa binaural e monoaural, não encontra diferença estatisticamente significante entre o tipo de aplicação quanto a variável idade e sexo. Conclui que o teste de limiar de fusão auditiva necessita ser estudado em trabalhos posteriores visto a grande influência que o processamento temporal tem no desenvolvimento dos aspectos de linguagem.
Resultados de várias pesquisas (Garcia, 2001; Chermak, 2002; Neijenhuis et al., 2001; Hautus, 2003; Moncrieff, 2004) indicam a necessidade de incluir na avaliação e/ou triagem do processamento auditivo os testes que avaliam os aspectos temporais.

Branco-Barreiro (2003) em seu estudo com crianças com ausência (grupo controle) e presença (grupo experimental) de dificuldade de leitura, utiliza o Randon Gap Detection Test (RGDT) para a verificação das habilidades auditivas de processamento temporal. As crianças do grupo controle apresentaram média de detecção de intervalo de tempo semelhante à do grupo experimental nas freqüências de 500, 1000 e $4000 \mathrm{~Hz}$. Já nas freqüências de $2000 \mathrm{~Hz}$ e na média os resultados apresentaram diferenças estatisticamente significantes, indicando que o grupo controle apresentou média de detecção de intervalo significantemente menor. Não houve diferença estatisticamente significante entre as quatro freqüências avaliadas e a comparação entre os grupos revelou diferença estatisticamente significante, em que o grupo controle apresentou média de detecção de intervalo de tempo significantemente menor. Como conclusão o teste RGDT mostrou-se bom para identificação de crianças com dificuldade de leitura.

Ziliotto et al. (2006), utilizando o teste RGDT, concluem que o critério de referência de normalidade para jovens e adultos para o limiar de acuidade temporal é de 10 milissegundos para o valor médio.

Conhecer a habilidade auditiva de resolução temporal em crianças nascidas pré-termo, pode contribuir para a elaboração de programas de intervenção para aprimorar o desenvolvimento de linguagem destes indivíduos.

Com este trabalho pretende-se mostrar a importância do teste RGDT, proposto por Keith (2000), como uma ferramenta para a avaliação da resolução temporal em crianças nascidas pré-termo, lembrando que este teste pode ser utilizado em indivíduos nascidos a termo ou não, a partir dos cinco anos de idade.

Este teste permite a detecção precoce de alteração dos processos temporais e essa identificação possibilita que haja uma intervenção para minimizar ou evitar futuros prejuízos de desenvolvimento de linguagem.

Sendo assim, o objetivo deste trabalho é comparar o comportamento auditivo de resolução temporal de crianças pré-escolares reunidas segundo o momento do nascimento ser pré-termo ou termo. 


\section{Método}

Este trabalho foi realizado com a aprovação do Comitê de Ética e Pesquisa da Universidade Federal de São Paulo - Hospital São Paulo sob o numero 0757/03. Pré-escolares com faixas etárias entre 5 e 6 anos, 11 meses e 29 dias e foram distribuídos em dois grupos: Grupo 1 composto por 44 crianças nascidas a termo, 20 do sexo feminino e 24 do sexo masculino e Grupo 2 composto por 26 crianças nascidas prétermo (tempo gestacional inferior a 37 semanas segundo a OMS - Organização das Nações Unidas), 12 do sexo feminino e 14 do sexo masculino. Os responsáveis assinaram um termo de consentimento livre e esclarecido e responderam a um questionário contendo ficha de identificação e perguntas acerca do desenvolvimento auditivo baseados na história clínica proposta por Pereira (1997) e fatores de riscos para audição proposto por Azevedo (1995).

Todos os participantes não apresentaram evidências de comprometimento neurológico. Os processos neuropsicolinguísticos não foram avaliados nesses pré-escolares selecionados.

Os participantes foram submetidos à audiometria tonal liminar, pesquisa do limiar de recepção de fala, imitânciometria e pesquisa de reflexo acústico. Para essa avaliação foram utilizados cabina portátil "São Luiz", audiômetro Maico MA41 com fone TDH 39e imitanciômetro Damplex A28. O audiômetro está calibrado segundo a norma IEC-NBR 60645 e o imitanciômetro segundo a norma ISO 389.

Foram incluídas as crianças cujos limiares para tom puro nas frequiências avaliadas de 250, 500, 1000, $2000,3000,4000,6000$ e $8000 \mathrm{~Hz}$ se mostrassem igual ou inferior a $15 \mathrm{~dB}$ NA. O limiar de reconhecimento de fala (LRF) foi estabelecido segundo a proposta de Redondo e Lopes Filho (1997). Foram selecionadas as crianças que apresentaram LRF compatível com os limiares tonais. A classificação da curva timpanométrica e da presença de reflexos acústicos foram os de Jerger conforme mencionado em Redondo e Lopes Filho (1997).

Em todos os participantes foi aplicado o teste RGDT. Este teste foi proposto por Keith (2000) e tem como objetivo identificar e quantificar a capacidade de um indivíduo em solucionar aspectos relacionados ao tempo do evento acústico. $\mathrm{O}$ nome dado ao comportamento auditivo que caracteriza este tipo de capacidade é resolução temporal. Em seu trabalho original, Keith (2000) encontrou um tempo de detecção de intervalo de tempo (gap detection) normal, igual ou menor a 20 milisegundos, resultado esse obtido pela média aritmética dos resultados obtidos para cada freqüência pré-estabelecida testada $(500,1000,2000$ e $4000 \mathrm{~Hz}$ ) em crianças normais.

Para esse teste foi utilizado um audiômetro Maico MA41 acoplado ao equipamento PAC 2002 produzidos pela acústica Orlandi (Indústria e Comércio de equipamentos e acessórios para Audiologia) que possibilita a realização de testes que necessitem dois canais.

O teste RGDT consiste em uma apresentação gravada de uma seqüência com nove estímulos sonoros com pequenos intervalos de tempo em que o indivíduo é instruído a responder verbalmente ao avaliador se está escutando um ou dois sons.

Para esse trabalho foram utilizados os subtestes 1 (treino) e o subteste 2 (padrão). As respostas foram registradas em folha própria (anexo 1).

O subteste 1 (treino) conta com estímulos de 500 $\mathrm{Hz}$ com intervalos de tempo entre 0 a 40 milissegundos distribuídos em ordem crescente. Após o treino iniciase o subteste 2 (padrão) com seqüências de nove estímulos na apresentação nas freqüências de 500, 1000,2000 e $4000 \mathrm{~Hz}$ com intervalos de tempo entre 0 e 40 milisegundos distribuídos aleatoriamente. Os intervalos apresentados são: 0, 2, 5, 10, 15, 20, 25, 30 e 40 milisegundos.

Todos os indivíduos foram treinados a ouvir em campo livre, antes da colocação do fone dentro da cabina e certificado da compreensão da realização do teste. Esse teste foi aplicado em diferentes situações que foram denominadas segundo a forma de apresentação de binaural (BI) e monoaural (MO). $\mathrm{Na}$ forma de apresentação monoaural foi utilizada a denominação à direita e à esquerda, procurando-se indicar a orelha que recebeu os estímulos isoladamente. Designou-se binaural por meio da abreviação BI, monoaural à direita por meio da abreviação MOD e monoaural à esquerda de MOE. Iniciou-se a avaliação pela apresentação binaural seguida da monoaural. Além disso, outra situação de teste, denominada de ordem de teste, envolveu a orelha que recebeu os estímulos inicialmente.

O teste RGDT foi apresentado a 40dB NS tendo por referência o limiar médio de audibilidade para 500,1000 e $2000 \mathrm{~Hz}$.

Os grupos foram subdivididos utilizando-se o critério de situação de aplicação do teste RGDT segundo a forma (apresentação binaural e monoaural) e segundo a ordem, ou seja, orelha que iniciou o teste na forma monoaural.

Desta forma as situações de aplicação do teste RGDT quanto à forma foram: apresentação binaural por freqüência $(500,1000,2000$ e $4000 \mathrm{~Hz})$ e 
apresentação monoaural por freqüência $(500,1000$, 2000 e $4000 \mathrm{~Hz}$ ). E, quanto à ordem de início do teste para a forma monoaural, foram: teste monoaural com início à orelha direita; teste monoaural com início à orelha esquerda. As crianças foram reunidas em grupo considerando cada uma dessas situações.

O primeiro grupo composto pelos indivíduos nascidos a termo (GT), que após realizarem o teste em sua apresentação binaural, o fizeram iniciando pela orelha direita e depois orelha esquerda para cada subteste, foi denominada abreviadamente como GTD. Esse grupo contou com 22 crianças.

O segundo grupo composto pelos indivíduos nascidos a termo (GT), que após realizarem o teste em sua apresentação binaural, o fizeram iniciando pela orelha esquerda e depois orelha direita para cada subteste, foi denominada abreviadamente como GTE. Esse grupo contou com 22 crianças.

O terceiro grupo composto pelos indivíduos nascidos pré-termo $(\mathrm{GPt})$, que após realizarem o teste em sua apresentação binaural, o fizeram iniciando pela orelha direita e depois orelha esquerda para cada subteste, foi denominada abreviadamente como GPtD. Esse grupo contou com 13 crianças.

O quarto grupo compostos pelos indivíduos nascidos pré-termo (GPt), que após realizarem o teste em sua apresentação binaural, o fizeram iniciando pela orelha esquerda e depois orelha direita para cada subteste, foi denominada abreviadamente como GPtE. Esse grupo contou com 13 crianças.

Foi realizado uma análise estatística descritiva dos dados de aplicação do teste RGDT por freqüência sonora utilizada considerando o gênero, a forma binaural e monoaural e ordem de inicio do teste (orelha direita primeira testada e orelha esquerda como primeira testada) e por grupo ( GTD, GTE e GPtD e GPtE).

Para a análise estatística desse trabalho foram utilizados testes não paramétricos: nas situações de dados pareados, foi utilizado o teste de Friedman, na presença de dados independentes o teste de Wilcoxon e quando se utilizou amostras independentes com o objetivo de comparar duasa-duas o teste utilizado foi de Mann Whitney . O nível de significância utilizado foi de 0,07 (7\%).

\section{Resultados}

Mostra-se na Tabela 1, utilizando o teste MannWhitney, os valores das medidas descritivas (mediana, média e seu respectivo desvio-padrão e os quartis) dos limiares de gap obtidos da aplicação do teste RGDT, na forma monoaural às orelhas direita e esquerda, e forma binaural por frequiência sonora, nos pré-escolares nascidos a termo dos Grupos GTD, GTE e total de nascidos a termo.

TABELA 1. Mediana, média e respectivo desvio-padrão, P-Valores e quartis, estabelecidos com base nas respostas dadas no teste RGDT em apresentação binaural e monoaural à orelha direita e esquerda, por frequiência pré-estabelecida obtidas pelos indivíduos do grupo de crianças nascidas a termo e seus sub-grupos: GTD e GTE.

\begin{tabular}{|c|c|c|c|c|c|c|c|c|c|}
\hline & \multicolumn{2}{|l|}{ GTD } & \multicolumn{2}{|c|}{ GTE } & \multicolumn{2}{|l|}{ GT } & \multirow{2}{*}{$\begin{array}{c}\text { P- Valor entre os } \\
\text { Grupos } \\
\text { GTD X GTE }\end{array}$} & \multirow{2}{*}{ Quartil 1} & \multirow{2}{*}{ Quartil 3} \\
\hline & Média $( \pm \mathrm{DP})$ & Mediana & Média $( \pm D P)$ & Mediana & Média & Mediana & & & \\
\hline 500 bin & $9,23( \pm 11,27)$ & 5 & $11,77( \pm 13,46)$ & 5 & $10,50( \pm 12,34)$ & 5 & 0,500 & 2 & 15 \\
\hline 1000 bin & $11,45( \pm 13,69)$ & 5 & $16,05( \pm 16,69)$ & 5 & $13,75( \pm 15,26)$ & 5 & 0,324 & 2 & 22,50 \\
\hline 2000 bin & $16,68( \pm 16,44)$ & 7,5 & $18,82( \pm 17,37)$ & 10 & $17,75( \pm 16,75)$ & 10 & 0,677 & 2 & 40 \\
\hline 4000 bin & $21,09( \pm 15,62)$ & 15 & $19,09( \pm 15,81)$ & 15 & $20,09( \pm 15,57)$ & 15 & 0,675 & 5 & 40 \\
\hline $500 \mathrm{OD}$ & $11,36( \pm 12,01)$ & 5 & $21,73( \pm 17,77)$ & 17,50 & ----- & ----- & --- & --- & --- \\
\hline $1000 \mathrm{OD}$ & $16,18( \pm 14,56)$ & 10 & $28,50( \pm 16,49)$ & 40 & ----- & ----- & ---- & ---- & --- \\
\hline 2000 OD & $18,23( \pm 13,32)$ & 12,5 & $24,95( \pm 16,08)$ & 27,5 & ----- & ----- & ---- & ---- & ---- \\
\hline $4000 \mathrm{OD}$ & $18,50( \pm 15,51)$ & 10 & $25,32( \pm 16,87)$ & 40 & ----- & ---- & ---- & --- & --- \\
\hline $500 \mathrm{OE}$ & $13,82( \pm 11,66)$ & 10 & $20,91( \pm 16,62)$ & 17,50 & ----- & ----- & ---- & --- & --- \\
\hline $1000 \mathrm{OE}$ & $14,59( \pm 13,89)$ & 7,5 & $25,45( \pm 16,03)$ & 35 & ----- & ----- & ---- & ---- & ---- \\
\hline $2000 \mathrm{OE}$ & $17,32( \pm 13,87)$ & 15 & $25,23( \pm 18,31)$ & 40 & ----- & ----- & ----- & ---- & ---- \\
\hline $4000 \mathrm{OE}$ & $18,64( \pm 13,11)$ & 12,5 & $28,05( \pm 16,65)$ & 40 & ---- & ---- & ---- & ---- & ---- \\
\hline
\end{tabular}

Legenda: GTD = grupo de crianças nascidas a termo que iniciou o teste pela orelha direita na apresentação monoaural; GTE = grupo de crianças nascidas a termo que iniciou o teste pela orelha esquerda na apresentação monoaural; GT = total de crianças nascidas a termo; DP = desvio-padrão. 
Apresenta-se na Tabela 2 os valores da mediana, média e o seu respectivo desvio-padrão e os quartis, utilizando o teste Mann-Whitney calculados para os resultados obtidos no teste RGDT na forma monoaural para as orelhas direita e esquerda, e forma binaural, por freqüência sonora , nos pré-escolares nascidos pré- termo dos Grupos GPtD, GPtE e total de nascidos pré-termo.

Apresenta-se na Tabela 3, o resultado do cálculo estatístico (Teste de Mann-Whitney) realizado para comparar cada situação de aplicação do teste, tais como, forma de apresentação - binaural, BI, e monoaural, MO, e ordem de apresentação - orelha direita como primeira testada e como segunda orelha testada levando em conta os valores obtidos para os subgrupos que iniciaram pela orelha direita, nascidos a termo GTD e nascidos pré-termo, o GPtD. Na Tabela 4, os mesmos cálculos são mostrados para os subgrupos que iniciaram a forma monoaural de aplicação do teste pela orelha esquerda , subgrupo nascido a termo GPE e nascido pré-termo GPtE.

TABELA 2. Mediana, médias e seu respectivos desvio-padrão, e quartis, estabelecidas com base nas respostas dadas no teste RGDT em apresentação binaural e monoaural à orelha direita e esquerda, por freqüência pré-estabelecida obtidas pelos indivíduos do grupo de crianças nascidas pré-termo e seus sub-grupos: GPtD e GPtE.

\begin{tabular}{|c|c|c|c|c|c|c|c|c|c|c|}
\hline \multicolumn{4}{|c|}{ GPtD } & \multicolumn{2}{|c|}{ GPtE } & \multicolumn{2}{|l|}{$\mathrm{GPt}$} & \multirow{2}{*}{$\begin{array}{c}\text { P- Valor Entre os } \\
\text { Grupos } \\
\text { GPtD X GPtE }\end{array}$} & \multirow{2}{*}{ Quartil 1} & \multirow{2}{*}{ Quartil 3} \\
\hline \multicolumn{2}{|c|}{ Média $( \pm \mathrm{DP})$} & \multicolumn{2}{|c|}{ Mediana } & Média ( $\pm \mathrm{DP})$ & Mediana & Média & Mediana & & & \\
\hline 500 bin & 25,69 & $7,14)$ & 40 & $22,15( \pm 17,99)$ & 20 & $23,92( \pm 17,31)$ & 32,50 & 0,543 & 5 & 40 \\
\hline 1000 bin & 25,46 & $7,82)$ & 40 & $22,69( \pm 19,47)$ & 40 & $24,08( \pm 18,34)$ & 40 & 0,691 & 2 & 40 \\
\hline 2000 bin & 31,31 & $3,80)$ & 40 & $30,31( \pm 16,12)$ & 40 & $30,81( \pm 14,71)$ & 40 & 0,904 & 26,25 & 40 \\
\hline 4000 bin & 28,85 & $2,77)$ & 40 & $29,92( \pm 16,31)$ & 40 & $29,38( \pm 14,71)$ & 40 & 0,725 & 16,25 & 40 \\
\hline $500 \mathrm{OD}$ & 30,00 & $2,42)$ & 40 & $31,92( \pm 15,35)$ & 40 & ----- & ---- & ---- & ---- & ---- \\
\hline $1000 \mathrm{OD}$ & 32,31 & $4,67)$ & 40 & $32,31( \pm 14,67)$ & 40 & ----- & ----- & ---- & ---- & ---- \\
\hline 2000 OD & 33,50 & $3,27)$ & 40 & $32,69( \pm 14,09)$ & 40 & ---- & ---- & ---- & ---- & --- \\
\hline $4000 \mathrm{OD}$ & 34,23 & $1,15)$ & 40 & $30,77( \pm 14,56)$ & 40 & ---- & ---- & --- & ---- & ---- \\
\hline $500 \mathrm{OE}$ & 30,00 & $3,84)$ & 40 & $30,54( \pm 15,15)$ & 40 & ----- & ----- & ---- & ---- & ---- \\
\hline $1000 \mathrm{OE}$ & 34,33 & $3,34)$ & 40 & $31,08( \pm 15,42)$ & 40 & ----- & ----- & ---- & ---- & ---- \\
\hline $2000 \mathrm{OE}$ & 34,33 & $3,34)$ & 40 & $31,85( \pm 15,61)$ & 40 & ---- & ----- & --- & ---- & --- \\
\hline $4000 \mathrm{OE}$ & 34,23 & $1,15)$ & 40 & $34,23( \pm 11,15)$ & 40 & ----- & ----- & ---- & ---- & ---- \\
\hline
\end{tabular}

Legenda: GPtD = grupo de crianças nascidas pré-termo que iniciaram o teste pela orelha direita na apresentação monoaural; GPtE = grupo de crianças nascidas pré- termo que iniciaram o teste pela orelha esquerda na apresentação monoaural; GPt = total de crianças nascidas prétermo; DP = desvio-padrão.

TABELA 3. P-valores calculados para comparação do resultado do teste RGDT entre os subgrupos termo e pré-termo que iniciaram o teste pela orelha direita considerando a apresentação binaural e a monoaural à orelha direita (primeira e segunda orelha testada).

\begin{tabular}{c|c|c|c}
\hline \multirow{2}{*}{ Freqüência $(\mathrm{Hz})$} & \multicolumn{3}{c}{ GTD X GPtD } \\
\cline { 2 - 4 } & BI & MO Orelha Direita = Primeira Orelha Testada & MO Orelha Esquerda = Segunda Orelha Testada \\
\hline 500 & $0,013^{*}$ & $<0,001^{*}$ & $0,003^{*}$ \\
1000 & $0,037^{*}$ & $0,007^{*}$ & $0,002^{*}$ \\
2000 & $0,017^{*}$ & $0,006^{*}$ & $0,003^{*}$ \\
4000 & $0,069^{*}$ & $0,003^{*}$ & $0,002^{*}$ \\
\hline
\end{tabular}

Legenda: BI = apresentação binaural; $\mathrm{MO}=$ apresentação monoaural; GTD = nascidos a termo que iniciaram o teste pela orelha direita na apresentação monoaural; GPtD = nascidos pré-termo que iniciaram o teste pela orelha direita na apresentação monoaural; $*$ = estatisticamente significante. 
TABELA 4. P-valores calculados para comparação do resultado do teste RGDT entre os subgrupos termo e pré-termo que iniciaram pela orelha esquerda considerando a apresentação binaural e a monoaural à orelha esquerda ( primeira e segunda orelha testada).

\begin{tabular}{c|c|c|c}
\hline \multirow{2}{*}{ Freqüência $(\mathrm{Hz})$} & \multicolumn{3}{|c}{ GTE x GPtE } \\
\cline { 2 - 4 } & BI & ME Orelha esquerda = Primeira Orelha Testada & MO Orelha Direita = Segunda Orelha Testada \\
\hline 500 & 0,181 & $0,074 \#$ & $0,077 \#$ \\
1000 & 0,404 & 0,363 & 0,406 \\
2000 & $0,078 \#$ & 0,273 & $0,103 \#$ \\
4000 & $0,071 \#$ & 0,262 & 0,306 \\
\hline
\end{tabular}

Legenda: $\mathrm{BI}=$ apresentação binaural; $\mathrm{MO}=$ apresentação monoaural; GTE = grupo de nascidos a termo que iniciaram o teste pela orelha esquerda na apresentação monoaural; GPtE = grupo de nascidos a pré-termo que iniciaram o teste pela orelha esquerda na apresentação monoaural; \# = tendência a significante.

$\mathrm{Na}$ Tabela 5 são apresentados os valores do cálculo estatístico (teste de Mann-Whitney) realizados para comparar os resultados obtidos para o teste RGDT por frequiência pré-estabelecida (500, 1000,2000 e $4000 \mathrm{~Hz}$ ), no grupo de crianças nascidas a termo, considerando a BI, MOD, MOE e a comparação dessas situações entre os resultados obtidos por gênero masculino e feminino.

Na Tabela 6, mostra-se os valores do cálculo estatístico (Teste de Mann-Whitney) realizado para comparar os resultados obtidos para o teste RGDT por freqüência pré-estabelecida $(500,1000,2000 \mathrm{e}$ $4000 \mathrm{~Hz}$ ), no grupo de crianças nascidas pré-termo considerando a forma BI, a forma MOD, a forma MOE e a comparação dessas situações entre os indivíduos do sexo masculino e feminino.

Mostra-se na Tabela 7 (Teste de Friedman e de Wilcoxon) os P-valores calculados para comparar os resultados obtidos para o teste RGDT por frequiência pré-estabelecidas (500, 1000, 2000 e 4000 $\mathrm{Hz}$ ), no grupo de crianças nascidas a termo, considerando a forma binaural (BI) e monoaural à direita (MOD) e monoaural à esquerda (MOE).

Apresenta-se na Tabela 8 os valores do cálculo estatístico (teste de Friedman e de Wilcoxon) realizado para comparar os resultados obtidos para o teste RGDT por freqüência pré-estabelecidas (500, 1000, 2000 e $4000 \mathrm{~Hz})$, no grupo de crianças nascidas pré-termo considerando a forma binaural (BI) e a orelha direita na forma monoaural (MOD) e orelha esquerda na forma monoaural (MOE).

Na Tabela 9 são mostrados os dados do intervalo de confiança para os limiares de gap obtidos por meio do teste RGDT no grupo de préescolares nascidos a termo. Ilustra-se esses dados na Figura 1.
TABELA 5. P-valores calculados para a comparação dos resultados do teste RGDT entre os pré-escolares nascidos a termo do sexo masculino e feminino levando em conta o subgrupo segundo a orelha que iniciou o teste na forma monoaural, a forma de apresentação binaural, monoaural à direita e monoaural à esquerda por frequência sonora pré-estabelecida.

\begin{tabular}{cc|c|c|c|c}
\hline \multicolumn{6}{c}{ Freqüências Sonoras Pré-Estabelecidas do Teste RGDT } \\
\hline \multirow{2}{*}{ BI } & & $500 \mathrm{~Hz}$ & $1000 \mathrm{~Hz}$ & $2000 \mathrm{~Hz}$ & $4000 \mathrm{~Hz}$ \\
\cline { 3 - 7 } & GTD & 0,970 & 1,000 & 0,700 & 0,482 \\
\multirow{2}{*}{ MOD } & GTE & 0,657 & 0,704 & 0,731 & 0,520 \\
\cline { 2 - 6 } & GTD & 0,279 & 0,676 & $0,109 \#$ & 0,288 \\
\cline { 2 - 6 } MOE & GTE & 0,508 & 0.939 & 0,314 & 0,942 \\
& GTD & $0,059 *$ & 0,398 & 0,121 & 0,459 \\
& GTE & 0,541 & 0,646 & 0,823 & 0,759 \\
\hline
\end{tabular}

GTD = grupo de crianças nascidas a termo que iniciaram o teste pela orelha direita na apresentação monoaural; GTE = grupo de crianças nascidas a termo que iniciaram o teste pela orelha esquerda na apresentação monoaural; MOD = orelha direita na apresentação monoaural; $\mathrm{MOE}=$ orelha esquerda na apresentação monoaural; BI = apresentação binuaral; * = estatisticamente signifcante; $\#=$ tendência a significante.

TABELA 6. P-valores calculados (teste de Mann-Whitney) para a comparação dos resultados do teste RGDT entre os pré-escolares nascidos pré-termo do sexo masculino e feminino levando em conta o subgrupo segundo a orelha que iniciou o teste na forma monoaural, a forma de apresentação binaural, monoaural à direita e monoaural à esquerda por frequiência sonora pré-estabelecida.

\begin{tabular}{ccccccc}
\hline \multicolumn{5}{c}{ Freqüências Sonoras Pré-Estabelecidas do Teste RGDT } \\
\hline \multirow{3}{*}{ BI } & & $500 \mathrm{~Hz}$ & $1000 \mathrm{~Hz}$ & $2000 \mathrm{~Hz}$ & $4000 \mathrm{~Hz}$ \\
& GPtD & 0,425 & 0,337 & 0,210 & 0,873 \\
& GPtE & $0,093 \#$ & 0,261 & $0,116 \#$ & $0,117 \#$ \\
\cline { 2 - 6 } MOD & GPtD & 0,151 & 0,197 & 0,265 & 0,276 \\
& GPtE & $0,040 *$ & $0,042 *$ & $0,042^{*}$ & $0,014 *$ \\
\cline { 2 - 6 } MOE & GPtD & $0,112 \#$ & 0,695 & 0,695 & 0,198 \\
& GPtE & 0,137 & $0,116 \#$ & $0,042^{*}$ & $0,042^{*}$ \\
\hline
\end{tabular}

$\mathrm{GPtD}=$ grupo de crianças nascidas pré-termo que iniciaram o teste pela orelha direita na apresentação monoaural; GPtE = grupo de crianças nascidas prétermo que iniciaram o teste pela orelha esquerda na apresentação monoaural; MOD = orelha direita na apresentação monoaural; MOE = orelha esquerda na apresentação monoaural; BI = apresentação binuaral; * = estatisticamente signifcante; \# = tendência a significante. 
TABELA 7. P-valor calculado para a comparação dos resultados do teste RGDT na forma de apresentação binaural, com orelha direita e orelha esquerda na forma monoaural levando em conta o grupo de pré-escolares nascidos a termo que iniciaram o teste pela orelha direita e pela orelha esquerda.

\begin{tabular}{cc|c|c|c|c}
\hline & & $500 \mathrm{~Hz}$ & $1000 \mathrm{~Hz}$ & $2000 \mathrm{~Hz}$ & $4000 \mathrm{HZ}$ \\
\hline BI X MOD X MOE & GTD & $\mathbf{0 , 0 4 2}^{*}$ & $\mathbf{0 , 0 2 6} *$ & 0,232 & 0,346 \\
Teste de Friedman & GTE & $\mathbf{0 , 0 1 1}^{*}$ & $\mathbf{0 , 0 0 3} *$ & 0,283 & 0,285 \\
\hline BI X MOD & GTD & 0,289 & $\mathbf{0 , 0 1 2} *$ & ----- & ----- \\
Teste de Wilcoxon & GTE & $\mathbf{0 , 0 1 1 *}$ & $\mathbf{0 , 0 0 2} *$ & ---- & ---- \\
\hline BI X MOE & GTD & $\mathbf{0 , 0 0 7 *}$ & $0,103 \#$ & ---- & ---- \\
Teste de Wilcoxon & GTE & $\mathbf{0 , 0 0 3 *}$ & $\mathbf{0 , 0 0 8} *$ & ---- & ---- \\
\hline MOD X MOE & GTD & 0,375 & 0,344 & ----- & ----- \\
Teste de Wilcoxon & GTE & 0,683 & 0,181 & ---- & ---- \\
\hline
\end{tabular}

GTD = grupo de crianças nascidas a termo que iniciaram o teste pela orelha direita na apresentação monoaural; GTE = grupo de crianças nascidas a termo que iniciaram o teste pela orelha esquerda na apresentação monoaural; MOD = orelha direita na apresentação monoaural; $\mathrm{MOE}=$ orelha esquerda na apresentação monoaural; BI = apresentação binuaral; * = estatisticamente signifcante; $\#$ = tendência a significante.

TABELA 8. P-valor calculado para a comparação do resultado do teste RGDT dos pré-escolares nascidos pré-termo que iniciaram o teste pela orelha direita e pela orelha esquerda, quanto a forma de apresentação binaural,, com orelha direita na forma monoaural e orelha esquerda na forma monoaural.

\begin{tabular}{cc|c|c|c|c}
\hline & & $500 \mathrm{~Hz}$ & $1000 \mathrm{~Hz}$ & $2000 \mathrm{~Hz}$ & $4000 \mathrm{HZ}$ \\
\hline $\begin{array}{c}\text { BI X MOD X MOE } \\
\text { teste de Friedman }\end{array}$ & GPtD & 0,260 & $\mathbf{0 , 0 2 2} *$ & 0,472 & $\mathbf{0 , 0 4 1}$ \\
\hline GIE & $\mathbf{0 , 0 0 4} *$ & $\mathbf{0 , 0 3 4} *$ & 0,174 & $\mathbf{0 , 0 4 7}$ \\
\hline teste de Wilcoxon & GPtE & $\mathbf{0 , 0 1 7} *$ & $\mathbf{0 , 0 3 9} *$ & ---- & 0,715 \\
\hline BI X MOE & GPtD & ------ & $\mathbf{0 , 0 6 8} *$ & ---- & $\mathbf{0 , 0 6 6} *$ \\
teste de Wilcoxon & GPtE & $\mathbf{0 , 0 2 7} *$ & $0,078 \#$ & ---- & $\mathbf{0 , 0 6 8} *$ \\
\hline MOD X MOE & GPtD & ------ & 0,655 & ---- & 1,000 \\
teste de Wilcoxon & GPtE & 0,593 & $0,102 \#$ & ----- & $\mathbf{0 , 0 4 9} *$ \\
\hline
\end{tabular}

$\mathrm{GPtD}=$ grupo de crianças nascidas pré-termo que iniciaram o teste pela orelha direita na apresentação monoaural; $\mathrm{GPtE}=$ grupo de crianças nascidas prétermo que iniciaram o teste pela orelha esquerda na apresentação monoaural; MOD = orelha direita na apresentação monoaural; MOE = orelha esquerda na apresentação monoaural; BI = apresentação binuaral; * = estatisticamente signifcante; $\#$ = tendência a significante.

\section{Discussão}

Antes de apresentar os comentários sobre os resultados propriamente ditos considera-se essencial mostrar alguns conceitos sobre o tema estudado.

O tempo é um dos componentes fundamentais de um sinal acústico e deve ser considerado na interpretação de uma informação transmitida auditivamente (Shinn, 2003). A habilidade auditiva de processamento temporal é um fator importante para a percepção de fala, contribuindo para a identificação de pequenos elementos fonéticos presentes no discurso e que alterações nessa habilidade auditiva sugere interferência na percepção de fala normal e reconhecimento dos fonemas. (Keith , 2000; Au e Lovegrove, 2001)

A audição binaural tem sua importância para a localização sonora, mas tem como principal benefício auxiliar na detecção de sons em ambientes ruidosos (Moore, 1991).

O processamento da informação temporal pode ocorrer monoauralmente e/ou binauralmente conforme já relatara Strouse et al. (1998). O processamento via monoaural relacionar-se-ia ao que ocorre com o sinal que chega a uma orelha (embora normalmente isso ocorresse paralelamente nas duas orelhas). O processamento via binaural relacionarse-ia à análise da diferença entre os sinais que chegam às duas orelhas. Porque esses processamentos ocorreriam com diferenças de tempo (monoaural em milissegundos e binaural frações de milissegundos), o processamento temporal monoaural estaria mais relacionado com o sinal de fala enquanto o processamento temporal binaural estaria mais relacionado com a separação do sinal com os sons competitivos.

No presente estudo, as diferenças de desempenho no teste RGDT encontradas entre as crianças nascidas a termo e pré-termo mostram uma diferença no comportamento auditivo da resolução temporal entre os grupos. Para os indivíduos nascidos a termo (Tabela 1), na forma de aplicação binaural do teste RGDT, não houve diferenças estatisticamente significantes entre os subgrupos GTD e GTE, e apesar da forma binaural mostrar limiares menores do que os encontrados na forma monoaural, as diferenças não foram estatisticamente significantes. O limiar de menor intervalo de tempo, gap detection, desta medida da acuidade temporal, não ultrapassou 15 milissegundos em mediana, e por freqüência sonora avaliada foi 5 a 10 milissegundos melhor para frequiências baixas $(500$ e $100 \mathrm{~Hz})$ do que para altas $(2000 \mathrm{e} 4000 \mathrm{~Hz})$ no grupo ouvintes nascidos a termo na forma de apresentação binaural. 
TABELA 9. Valores do limite inferior, média e limite superior que compõem o intervalo de confiança para os limiares de gap em milisegundos obtidos por meio do teste RGDT em pré-escolares nascidos a termo (GT).

\begin{tabular}{cccccc}
\hline \multicolumn{2}{c}{ GT } & & Limite Inferior & Média & Limite Superior \\
\hline \multirow{3}{*}{ binaural } & $500 \mathrm{~Hz}$ & 6,86 & 10,5 & 14,14 \\
& $1 \mathrm{KHz}$ & 9,24 & 13,75 & 18,26 \\
& $2 \mathrm{KHz}$ & 12,8 & 17,75 & 22,7 \\
& $4 \mathrm{KHz}$ & 15,49 & 20,09 & 24,69 \\
\hline \multirow{2}{*}{ monoaural } & $500 \mathrm{~Hz}$ & 11,67 & 16,14 & 20,6 \\
orelha direita & $1 \mathrm{KHz}$ & 16,14 & 20,82 & 25,5 \\
& $2 \mathrm{KHz}$ & 16,94 & 21,73 & 26,52 \\
& $4 \mathrm{KHz}$ & 18,36 & 23,27 & 28,18 \\
\hline \multirow{2}{*}{ monoaural } & $500 \mathrm{~Hz}$ & 13,23 & 17,77 & 22,32 \\
orelha esquerda & $1 \mathrm{KHz}$ & 16,63 & 21,55 & 26,46 \\
& $2 \mathrm{KHz}$ & 16,6 & 21,14 & 25,67 \\
& $4 \mathrm{KHz}$ & 17,45 & 21,98 & 26,5 \\
\hline
\end{tabular}

Legenda: GT = grupo total de crianças nascidos a termo.

FIGURA 1. Intervalo de confiança para os limiares de gap em milisegundos obtidos por meio do teste RGDT, na apresentação binaural, e monoaural a orelha direita e esquerda no grupo de pré-escolares nascidos a termo.

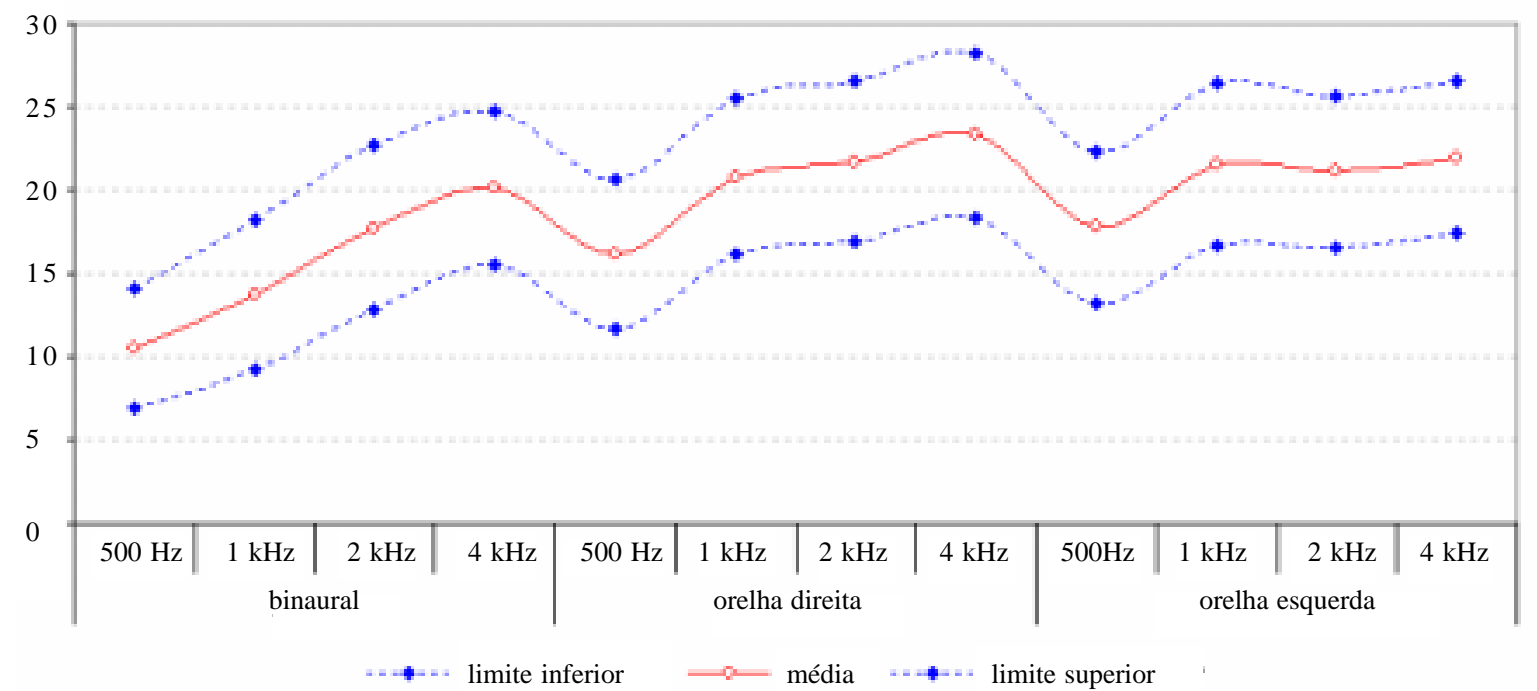

Nos indivíduos pré-termo (Tabela 2), a mediana do limiar de gap na forma de aplicação binaural e monoaural esteve em torno de 40 milissegundos. Em todas as situações de teste, o grupo pré-termo possuiu limiares de detecção de intervalo maiores do que o grupo termo.

$\mathrm{Na}$ forma binaural e na forma monoaural quando o teste iniciou pela orelha direita as diferenças entre os resultados das crianças nascidas a termo e prétermo foram estatisticamente significantes (Tabela 3). Quando o teste foi realizado monoauralmente iniciando pela orelha esquerda, as maiores médias continuaram pertencendo ao grupo nascido prematuramente, porém sem diferença estatisticamente significante (Tabela 4).

Nos trabalhos brasileiros compulsados, Garcia (2001) e Costa (2002) utilizando o teste AFT-R e AFT ( Auditory Fusion Test - Revised e Auditory Fusion Test) encontraram diferenças estatisticamente significantes entre a aplicação binaural e monoaural. Cabe destacar que nesses estudos foi considerado o limiar de fusão da acuidade auditiva que é diferente do limiar do intervalo de tempo, gap detection, que foi avaliado nesta pesquisa.

Analisando a variável gênero no grupo de préescolares nascidos a termo (Tabela 5) verificou-se em apenas uma situação, no grupo GTD e tendo a forma monoaural de apresentação do teste para a 
orelha esquerda, que somente na freqüência de $500 \mathrm{~Hz}$ houve diferença estatisticamente significante. Nas demais situações não houve diferença estatisticamente significante em qualquer forma de apresentação do teste (binaural e monoaural) considerando as freqüências préestabelecidas do teste.

Mais uma vez, enfocando a variável gênero no grupo de pré-escolares nascidos pré-termo (Tabela 6) não houve relevância estatística na forma de apresentação binaural. Já na forma monoaural verificou-se diferenças estatisticamente significantes principalmente no grupo que iniciou o teste pela orelha esquerda GPtE.

Garcia (2001) e Branco-Barreiro (2003) não incluíram a variável gênero em suas pesquisas. Costa (2002) em seu estudo não encontrou diferença estatisticamente significante entre os resultados quanto a variável gênero, porém os limiares encontrados no sexo masculino foram menores em relação ao sexo feminino, fato esse também presenciado nesse estudo.

No estudo das comparações dos resultados do sub-grupo GTD quanto às formas de apresentação (binaural x monoaural) do teste RGDT em suas freqüências sonoras pré-estabelecidas, (Tabela 7) verificou-se que os limiares de detecção de intervalo de tempo foram menores (Tabela 1), estatisticamente significantes (Tabela 7), na apresentação binaural nas frequiências de $500 \mathrm{~Hz}$ e de $1000 \mathrm{~Hz}$ quando comparados à apresentação monoaural. As freqüências de 2000 e 4000 Hz não apresentaram diferenças estatisticamente significantes.

No sub-grupo GTE, as comparações dos resultados quanto às formas de apresentação do teste RGDT em suas freqüências sonora préestabelecidas (Tabela 7) mostraram que as frequiências de 500 e $1000 \mathrm{~Hz}$ na forma binaural apresentaram diferenças estatisticamente significantes quando comparadas com a apresentação monoaural: os limiares de detecção de intervalo de tempo binaurais foram menores à esquerda e à direita em ambas as freqüências. As freqüências de 2000 e $4000 \mathrm{~Hz}$ não apresentaram diferenças estatisticamente significantes.

Os resultados quanto às combinações entre as formas de apresentação do teste RGDT em suas freqüências pré-estabelecidas (Tabela 8) no subgrupo GPtD, mostraram diferenças estatisticamente significantes quando se comparou a apresentação binaural com a monoaural. Os limiares de detecção de intervalo de tempo foram maiores em 1000 e $4000 \mathrm{~Hz}$ à esquerda e em $4000 \mathrm{~Hz}$ à direita do que os obtidos na forma binaural. Nas freqüências de $500 \mathrm{e}$ $2000 \mathrm{~Hz}$ não houve diferenças estatisticamente significantes. No sub-grupo GPtE, observou-se diferenças estatisticamente significantes (Tabela 8) quando se comparou a apresentação binaural com a monoaural nas freqüências de 500,1000 e 4000Hz. Os limiares da apresentação binaural foram menores do que os obtidos na forma monoaural (Tabela 2).

Garcia (2001) não encontrou diferenças estatisticamente significantes entre as respostas levando em conta a orelha em que o estímulo foi apresentado, orelha direita e orelha esquerda, nas freqüências testadas de 1000 e $4000 \mathrm{~Hz}$.

O intervalo de confiança dos resultados para o teste RGDT na sua forma binaural e monoaural (Tabela 9 e figura1) mostram que os limiares de gap não ultrapassam 20 milisegundos na forma binaural nas freqüências sonoras de 500,1000 e não ultrapassam 25 milissegundos em 2000 e $4000 \mathrm{~Hz}$. $\mathrm{Na}$ forma monoaural esses limites são aumentados em torno de 5 a 10 milissegundos.

Sendo assim, recomenda-se que o teste RGDT seja aplicado na forma binaural pois parece ser a mais fácil, mais rápida e a que apresentou menores limiares neste estudo. Segundo essa pesquisa, as crianças nascidas pré-termo apresentaram uma habilidade de resolução temporal inferior às nascidas a termo, evidenciada principalmente na forma binaural de obtenção do limiar de gap. Na medida em que o teste RGDT for utilizado para novos estudos, esses resultados devem ser comparados.

$\mathrm{Na}$ literatura especializada compulsada vários autores (Dupin et al., 2000; Davis et al., 2001; Demanez et al., 2003) relatam em seus estudos desempenho inferior no comportamento auditivo em nascidos prétermo quando comparados com nascidos a termo.

Sabe-se que a inabilidade de resolução temporal diz respeito a dificuldade de perceber estímulos que se modificam rapidamente e isso pode afetar o processamento fonológico dos sons da língua , a discriminação desses sons, e assim pode interferir com a compreensão da fala principalmente em velocidade aumentada. Com base nos achados neste trabalho acredita-se que as crianças nascidas prétermo podem ser consideradas de risco para apresentar alterações de linguagem, de leitura e de aprendizagem uma vez que seu desempenho na habilidade de resolução temporal foi inferior as crianças da mesma faixa etária nascidas a termo.

Sendo assim, recomenda-se que seja realizada uma avaliação quanto ao comportamento de resolução temporal em crianças nascidas pré-termo, possibilitando quando necessário, um trabalho de intervenção fonoaudiológica para minimizar dificuldades no desenvolvimento de audição e de linguagem que possivelmente possam ocorrer. 


\section{Conclusão}

As crianças nascidas pré-termo apresentaram uma habilidade de resolução temporal inferior às nascidas a termo, evidenciada principalmente na forma binaural de obtenção do limiar de gap entre tons puros de diferentes freqüências sonoras.

\section{Referências Bibliográficas}

AMERICAN SPEECH-LANGUAGE-HEARING ASSOCIATION (ASHA). Central auditory processing: current status of research and implications for clinical practice. Am. J. Audiol., v. 5, p. 41-54, 1996.

AU, A.; LOVEGROVE, B. Temporal processing ability in above average and average readers. Percept. Psychophys., v. 63, n. 1, p. 148-155, 2001.

AZEVEDO, M. F.; VIEIRA; R. M.; VILANOVA; L. C. Desenvolvimento auditivo de crianças normais $e$ de alto risco. São Paulo: Plexus, 1995.

BAILEY, P. J.; SNOWLING, M. J. Auditory processing and the development of language and literacy. Br. Med. Bull., v. 63, p. 135-146, 2002.

BRANCO-BARREIRO, F. C. A. Estudo do processamento auditivo temporal em alunos de escola pública com e sem dificuldade de leitura. 2003. Tese (Doutorado) Universidade de São Paulo, São Paulo.

BREIR, J. I.; FLETCHER, J. M.; FOORMAN, B. R.; KLAAS, P.; GRAY, L. C. Auditory temporal processing in children with specific reading disability with and without attention deficit/hyperactivity disorder. J. Speech Lang. Hear. Res., v. 46, p. 31-42, 2003.

CESTNICK, L.; JERGER, J. Auditory temporal processing and lexical/nonlexical reading in developmental dyslexics. J. Am. Acad. Audiol., v. 11, n. 9, p. 501-513, 2000.

CHERMAK, G. D. Deciphering auditory processing disorders in children. Otolaryngol. Clin. North Am., v. 35, n. 4, p. 733-749, 2002.

COSTA, L. A. Teste de fusão auditiva: estudo em crianças escolares. 2002. Monografia (Especialização) Universidade Federal de São Paulo, São Paulo.

DAVIS, N. M.; DOYLE, L. W.; FORD, G. W.; KEIR, E.; MICHAEL, J.; RICKARDS, A. L.; KELLY, E. A.; CALLANAN, C. Auditory function at 14 years of age of very-low-birthweight children. Dev. Med. Child Neurol., v. 43, p. 191-196, 2001.

DEMANEZ, L.; BONIVER, V.; DONY-CLOSON, B.; LHONNEUX-LEDOUX, F.; DEMANEZ, J. P. Central auditory processing disorders: some cohorts studies. Acta Otorhinolaryngol. Belg., v. 57, p. 291-299, 2003.

DUPIN, R.; LAURENT, J. P.; STAUDER, J. E. A.; SALIBA, E. Auditory attention processing in 5-year-old children born preterm: evidence from event-related potentials. Dev Med Child Neurol., v. 42, p. 476-480, 2000 .
GARCIA, V. L. Processamento auditivo em crianças com e sem distúrbios de aprendizagem. 2001. $298 \mathrm{f}$. Dissertação (Doutorado em Distúrbios da Comunicação Humana) - Universidade Federal de São Paulo, São Paulo.

HAUTUS, M. J.; SETCHELL, G. J.; WALDIE, K. E.; KIRK, I. J. Age-related improvements in auditory temporal resolution in reading-impaired children. Dyslexia, v. 9, n. 1, p. 37-45, 2003.

JERGER, J.; MUSIEK, F. Report of consensus conference on the diagnosis of auditory processing disorders in schoolaged children. J. Am. Acad. Audiol., v. 11, p. 467-474, 2000 .

KEITH, R. W. Random gap detection test. Missouri (USA): Auditec of Saint Louis, 2000.

MONCRIEFF, D. Temporal processing deficits in children with dyslexia. [S.n., s.1], apr. 2004. Disponível em: <http:/ / w w w . a u d o log y o n line.com/articles / arc_disp.asp?id=725\&catid=11>. Acesso em: 25 jul. 2004.

MOORE, D. R. Anatomy and physiology of binaural hearing. Audiol., v. 30, p. 125-134, 1991.

NEIJENHUIS, K. A. M.; STOLLMAN, M. H. P.; SNIK, A. F. M.; BROEK, P. V. D. Development of a central auditory test battery for adults. Audiol., v. 40, p. 69-77, 2001.

PEREIRA, L. D.; SCHOCHAT, E. Processamento auditivo central: manual de avaliação. São Paulo: Lovise, 1997.

PHILLIPS, S. L.; GORDON-SALANT, S.; FITZGIBBONS, P. J.; YENI-KOMSHIAN, G. Frequency and temporal resolution in elderly listeners with good and poor word recognition. J. Speech Lang. Hear. Res., v. 43, n. 1, p. 217-228, 2000.

REDONDO, M. C.; LOPES, O. C. F. [S.n.]. In: LOPES, O. F. (Edit.). Tratado de Fonoaudiologia. São Paulo: Roca, 1997.

SHINN, J. B. Temporal processing: the basics. Hear. J., v. 56, n. 7, p. 52, 2003.

Strouse, A.; ASHMEAD, D. H.; OHDE, R. N.; GRANTHAM, D. W. Temporal processing in the aging auditory sistem. J. Acoust. Soc. Am., v. 104, n. 4, p. 2385, 1998. 